\title{
Tensor-Based Morphometry Reveals Volumetric Deficits in Moderate/Severe Pediatric Traumatic Brain Injury
}

\author{
Emily L. Dennis, Xue Hua,, Julio Villalon-Reina, Lisa M. Moran, ${ }^{2}$ Claudia Kernan, ${ }^{2}$ \\ Talin Babikian, ${ }^{2}$ Richard Mink, ${ }^{3}$ Christopher Babbitt, ${ }^{4}$ Jeffrey Johnson, \\ Christopher C. Giza, ${ }^{6}$ Paul M. Thompson, ${ }^{1,7}$ and Robert F. Asarnow ${ }^{2,8}$
}

\begin{abstract}
Traumatic brain injury (TBI) can cause widespread and prolonged brain degeneration. TBI can affect cognitive function and brain integrity for many years after injury, often with lasting effects in children, whose brains are still immature. Although TBI varies in how it affects different individuals, image analysis methods such as tensor-based morphometry (TBM) can reveal common areas of brain atrophy on magnetic resonance imaging (MRI), secondary effects of the initial injury, which will differ between subjects. Here we studied 36 pediatric moderate to severe TBI (msTBI) participants in the post-acute phase (1-6 months post-injury) and $18 \mathrm{msTBI}$ participants who returned for their chronic assessment, along with well-matched controls at both time-points. Participants completed a battery of cognitive tests that we used to create a global cognitive performance score. Using TBM, we created three-dimensional (3D) maps of individual and group differences in regional brain volumes. At both the post-acute and chronic time-points, the greatest group differences were expansion of the lateral ventricles and reduction of the lingual gyrus in the TBI group. We found a number of smaller clusters of volume reduction in the cingulate gyrus, thalamus, and fusiform gyrus, and throughout the frontal, temporal, and parietal cortices. Additionally, we found extensive associations between our cognitive performance measure and regional brain volume. Our results indicate a pattern of atrophy still detectable 1-year post-injury, which may partially underlie the cognitive deficits frequently found in TBI.
\end{abstract}

Key words: MRI; pediatric; tensor based morphometry; traumatic brain injury

\section{Introduction}

T RAUMATIC BRAIN INJURY (TBI) is a major public health issue and the leading cause of death and disability in children and adolescents, leading to more than 500,000 emergency room visits per year in the United States alone, according to the American Academy of Pediatrics. ${ }^{1}$ Children are especially vulnerable to TBI, and TBI is more prevalent in children than in adults, often causing long-term damage to the developing brain. Brain maturation is a long process, and growth occurs at different rates and in different patterns across the brain. ${ }^{2,3}$ The sudden and devastating shock of a TBI can disrupt the normal developmental trajectory of the brain and cognitive functions. Here we examined the effects of moderate to severe TBI (msTBI) on regional brain volume in pediatric patients, and whether those effects are linked to the cognitive deficits well documented following TBI.

In msTBI there is a considerable amount of heterogeneity that can affect analyses. Patients with msTBI vary in their severity, type, and location of injury, and in pre-existing conditions, as well as numerous other factors that can affect recovery, including age and co-morbid conditions. Nevertheless, group analyses have revealed typical patterns of volume reductions across a number of subcortical and cortical regions for an extended period of time postinjury. The corpus callosum (CC); corona radiata; anterior, mid, and posterior cingulate gyrus; precuneus; and parahippocampal gyrus all show lower mean volumes in TBI in adults. ${ }^{4-9}$ Prior research in both adults and pediatric patients has found volume reductions in many subcortical areas, including the hippocampus,

\footnotetext{
${ }^{1}$ Imaging Genetics Center, Keck School of Medicine; ${ }^{7}$ Departments of Neurology, Pediatrics, Psychiatry, Radiology, Engineering, and Ophthalmology, USC, Marina del Rey, California.

${ }^{2}$ Department of Psychiatry and Biobehavioral Sciences, Semel Institute for Neuroscience and Human Behavior; ${ }^{8}$ Department of Psychology, UCLA, Los Angeles, California.

${ }^{3}$ Harbor-UCLA Medical Center and Los Angeles BioMedical Research Institute, Department of Pediatrics, Torrance, California.

${ }^{4}$ Miller Children's Hospital, Long Beach, California.

${ }^{5}$ LAC+USC Medical Center, Department of Pediatrics, Los Angeles, California.

${ }^{6}$ UCLA Brain Injury Research Center, Dept of Neurosurgery and Division of Pediatric Neurology, Mattel Children's Hospital, Los Angeles, California.
}

(C) Emily L. Dennis et al. 2015; Published by Mary Ann Liebert, Inc. This Open Access article is distributed under the terms of the Creative Commons Attribution Noncommercial License (http://creativecommons.org/licenses/by-nc/4.0/) which permits any noncommercial use, distribution, and reproduction in any medium, provided the original author(s) and the source are credited. 
amygdala, putamen, globus pallidus, thalamus, caudate, and midbrain. ${ }^{4,6-8,10,11}$ Along with general volume reductions found across the frontal, temporal, and parietal lobes, ventricular enlargement has been found in adult TBI patients. ${ }^{6-9,12,13}$ In children, more severe TBI is associated with smaller volumes in prefrontal and temporal cortices, as well as the $\mathrm{CC} .^{14-18}$ Injuries requiring longer intensive care have been associated with smaller head circumference in pediatric patients. ${ }^{10}$ The cerebellum, cerebellar peduncles, and brainstem are also susceptible to $\mathrm{TBI},{ }^{4,6,8}$ and functional abnormalities in these regions may partially underlie deficits in processing speed. ${ }^{6}$

Several studies have reported morphometric differences in pediatric TBI patients, ${ }^{10,11,14-18}$ and other studies have used tensorbased morphometry (TBM) to investigate TBI in adult patients. ${ }^{4,6,7}$ To the authors' knowledge, no prior studies have applied TBM to pediatric TBI. TBM relies on deformation fields describing the relative positions of various brain structures, comparing each individual's brain scan to an average brain template. These deformation fields are used to create images indicating regional volume expansion or contraction, relative to the template. The advantage of TBM is that it assesses the whole brain at once, without requiring $a$ priori hypotheses on the most likely locations of the group differences. An additional advantage of TBM over other related methods such as voxel-based morphometry (VBM), is that it does not rely on accurate gray/white matter (WM) tissue segmentation, which can be less reliable in injured brains. The deformation fields that TBM generates can give an accurate estimate of volume change, even when there are extensive brain tissue deformations caused by TBI.

We examined a large cohort of pediatric msTBI patients at two distinct, circumscribed time points post-injury. In the "post-acute" phase (1-6 months post-injury), we examined 36 msTBI participants $(8-19$ years old, mean age $=14.1$ years, standard deviation $[\mathrm{SD}]=2.7,26$ male $[\mathrm{M}] / 10$ female $[\mathrm{F}]$ ), and in the chronic phase (13-19 months post-injury), we examined $18 \mathrm{msTBI}$ participants (mean age $=15.9$ years, $\mathrm{SD}=2.6,13 \mathrm{M} / 5 \mathrm{~F}$ ), along with wellmatched controls in each phase. We used TBM to identify regional differences in brain volume. We hypothesized that the msTBI group would show volume reductions, especially in the $\mathrm{CC}$ and subcortical regions, and that these volume reductions would persist from the post-acute phase to the chronic phase. We also hypothesized that deficits in regional brain volume at both time-points would be associated with deficits in cognitive function.

\section{Methods}

\section{Participants}

TBI participants were recruited from four pediatric intensive care units (PICUs) located in Level 1 trauma centers in Los Angeles County. In these institutions, patients with msTBI are routinely admitted to the PICU. A study representative discussed the goals of the study with the parents of patients, gave them an institutional review board-approved brochure about the study, and obtained permission for the investigators to contact them after discharge from the medical center. Thirty-five percent of patients whose parents agreed to be contacted while the child was in the PICU participated in this study. Of 124 families contacted at the PICUs, 27 were lost to contact (kept canceling/rescheduling), 21 did not qualify because they did not meet criteria (Glasgow Coma Scale [GCS] score ${ }^{19}>12$, English skills not sufficient, attention deficit hyperactivity disorder [ADHD], learning disability, braces, etc.), 26 were not interested, 48 are participating, and 2 are interested, pending scheduling. Healthy controls, matched for age, sex, and educational level, were recruited from the community through flyers, magazines, and school postings. Several participants were only scanned in the chronic phase, whereas a few additional participants scanned in the post-acute phase (not included in the numbers above) were scanned outside our established 1-6 month time window, so were not included in this analysis. For those participants who completed the longitudinal assessment, the chronic assessment was completed approximately 12 months after the post-acute assessment. Demographics of the participants included in cross-sectional analyses of the post-acute and chronic phases are presented in Table 1. The injury mechanisms for our TBI group were as follows, out of 36 total injured participants: 12 motor vehicle accident (MVA) struck as a pedestrian, 6 MVA-passenger, 7 skateboard, 3 scooter, 2 bike, 1 blunt head trauma from sports, 1 assault, 1 skiing, 1 fall from ladder, 1 uncategorized blunt head trauma, and 1 uncategorized skull fracture. The demographic information from our sample is consistent with the existing epidemiological information on moderate-severe pediatric/adolescent TBI, both in the male to female ratio and in the types of mechanisms of injury. ${ }^{20}$ In our post-acute sample, the average GCS score (initial GCS upon hospital admission) was 8.75 ( $\mathrm{SD}=3.7), 18 \%$ of participants had neurosurgery, and $18 \%$ had evidence of increased intracranial pressure (ICP) on computed tomography (CT). In our chronic sample, the average GCS score (initial GCS upon hospital admission) was $9.33(\mathrm{SD}=3.8), 38 \%$ participants had neurosurgery, and $19 \%$ had evidence of increased ICP on CT.

In this study, we focus on cross-sectional analyses. Longitudinal analyses will be a focus of future studies as children continue to return for their follow-up visits. Thirty-three of the participants in the chronic phase completed the post-acute assessment prior. Seven of the chronic participants were not enrolled in time for a post-acute assessment, or did not complete magnetic resonance imaging (MRI) at time 1. The majority of participants in the post-acute phase have returned or will return for their time 2 follow-up, but several participants have not continued in the study, as detailed in our earlier article. ${ }^{21}$ The most frequent reasons for leaving the study were that the participants no longer met criteria or moved out of the area; there was no systematic bias introduced through these non-returns.

Inclusion criteria. 1) non-penetrating msTBI (intake or postresuscitation GCS score between 3 and 12);2) 8-19 years of age; 3) right-handed; 4) normal visual acuity or vision corrected with contact lenses/eyeglasses; and 5) English skills sufficient to understand instructions and be familiar with common words (the neuropsychological tests used in this study presume competence in English).

Exclusion criteria. 1) history of neurological illness, such as prior msTBI, brain tumor, or severe seizures; 2) motor deficits that

Table 1. Demographic Information

\begin{tabular}{lccccc}
\hline & \multicolumn{2}{c}{ Post-acute } & & \multicolumn{2}{c}{ Chronic } \\
\cline { 2 - 3 } \cline { 5 - 6 } \cline { 5 - 6 } & TBI & Control & & TBI & Control \\
\hline $\mathrm{N}$ & 36 & 35 & & 18 & 22 \\
$\mathrm{Age}$ & $14.1(2.7)$ & $14.8(2.8)$ & & $15.8(2.6)$ & $16.2(3.2)$ \\
$\mathrm{M} / \mathrm{F}$ & $26 / 10$ & $23 / 12$ & & $13 / 5$ & $15 / 7$ \\
$\mathrm{ICV}\left(\mathrm{cm}^{3}\right)$ & $1940(63)$ & $1945(68)$ & & $1957(82)$ & $1925(62)$ \\
\hline
\end{tabular}

Demographic information on the participants. Information is listed for both the post-acute phase (1-6 months post-injury), and the chronic phase (13-19 months post-injury). We list the number of TBI and control participants in each phase, the male/female ratio, the average age of these groups (and standard deviation), and the ICV (intracranial volume, in $\mathrm{cm}^{3}$ ).

TBI, traumatic brain injury. 
prevent the subject from being examined in an MRI scanner (e.g., spasms); 3) history of psychosis, ADHD, Tourette's syndrome, learning disability, mental retardation, autism, or substance abuse. These conditions were identified by parental report and are associated with cognitive impairments that might overlap with those caused by TBI. Participants were excluded if they had metal implants that prevented them from safely undergoing an MRI scan.

\section{Scan acquisition}

Participants were scanned on 3T Siemens Trio MRI scanners (Siemens Healthcare GmbH, Germany) with magnetizationprepared rapid gradient echo imaging (MPRAGE). The T1weighted images were acquired with the following acquisition parameters: GRAPPA mode; acceleration factor phase encoding $(\mathrm{PE})=2$; repetition time $(\mathrm{TR}) / \mathrm{echo}$ time $(\mathrm{TE}) / \mathrm{T} 1=1900 / 3.26 /$ $900 \mathrm{msec}$; field of view $(\mathrm{FOV})=250 \times 250 \mathrm{~mm}$; an axial plane acquisition with isotropic voxel size $=1 \mathrm{~mm}$, flip angle $=9$ degrees.

\section{Scan comparison}

Partway through the study, scanning moved from the UCLA Brain Mapping Center (BMC) to the Staglin IMHRO Center for Cognitive Neuroscience (Staglin). Both scanners were 3T Siemens Trio scanners, and the protocol was maintained. In the post-acute phase, 29 participants were scanned at BMC and 42 at Staglin. In the chronic phase, 9 participants were scanned at BMC and 31 at Staglin. To determine that this scanner change did not introduce bias into our data, we scanned 6 healthy adult volunteers at both the BMC and Staglin centers, 1.5 months apart. We then assessed possible scanner effects based on the T1-weighted image pairs. Extensive details of this process can be found in our prior article. ${ }^{21}$ For the T1-weighted images, scan comparisons revealed no detectable pattern in the difference between the intensity correction fields above noise, except in the cerebellum, where there appeared to be scanner-induced differences in image intensity even after N3 (intensity non-uniformity) correction (http://www.itksnap.org). For this reason, the cerebellum was masked out of the analyses presented in this article.

\section{Cognitive measures}

Participants completed a battery of cognitive tests at both time 1 and time 2. To capture overall ability, a performance index was generated based on the linear combination of the following agebased standardized or scaled measures: 1) Processing Speed Index from the Wechsler Intelligence Scale for Children, 4th ed. (WISCIV)/Wechsler Adult Intelligence Scale, 3rd ed. (WAIS-III) ${ }^{22} ; 2$ ) Working Memory Index from the WISC-IV/WAIS-III ${ }^{22} ; 3$ ) Trials $1-$ 5 from the California Verbal Learning Test-Children's Version, 2nd ed.

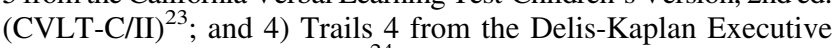
Function System (D-KEFS). ${ }^{24}$ These measures assess cognitive domains most sensitive to the effects of TBI. ${ }^{25}$ The WISC and WAIS-based Processing Speed Index comprised the Coding and Symbol Search subtests. For the Working Memory Index, the Digit Span and the Letter-Number Sequencing subtests were included. The CVLT (child or adult version) Trials $1-5$ score assesses verbal learning and short-term memory. Condition 4 of the Trail Making Test was a measure of switching attention. A series of principal components analyses confirmed these measures share sufficient common variance in both children with TBI and healthy controls.

\section{Tensor-based morphometry}

Each subject's T1-weighted anatomical data was N3-corrected using c3d (http://www.itksnap.org) to correct for intensity inhomogeneities. Volumes were then automatically skull-stripped using Brainsuite (http://brainsuite.org) and these masks were manually edited by a trained neuroanatomical expert (ELD and JVR). We used a study-specific registration template, motivated by previous studies finding that this leads to better registration results. We chose a healthy control male, aged 14.2 years (the average for our sample at time 1) with a visually normal T1-weighted scan to initialize the linear registration. This examplar subject was registered to the international consortium for brain mapping (ICBM) template using the fsl tool flirt (http://fsl.fmrib.ox.ac.uk), using 7 degrees of freedom (DOF) registration, with trilinear interpolation, and using mutual information as the similarity function for alignment. Following this, each subject's masked, non-uniformity (NU)-corrected T1-weighted image was registered to the subject-template using iterative 6,7 , and 9 DOF registration, with concatenated transformation files so that only one resampling step was run. This protocol was modified for this dataset from the original protocol. ${ }^{26,27}$

At this point, we created a minimal deformation template (MDT) from the healthy controls (19 female/23 male). The MDT is the template that deviates least from the anatomy of the subjects, with respect to a mathematically defined metric of difference, and, in some circumstances, using a MDT can improve statistical power. ${ }^{28}$ The MDT serves as an unbiased registration target for non-linear registrations.

Next, each subject's aligned T1s were non-linearly aligned to the MDT template, using an inverse consistent elastic-model registration algorithm (32 fast fourier transform [FFT] $\times 300$ iterations). ${ }^{29}$ The inter-subject displacement vector field, obtained from this step, was then applied to transform the Jacobian map of each subject to the brain coordinates defined by the MDT. Spatial normalizations among different brains enable regional comparisons and intergroup statistical analyses.

\section{Group comparison}

We ran voxel-wise linear regression testing for group differences, including age, sex, scanner, and intracranial volume (ICV) as covariates. The 9 DOF linear registration that is part of our processing protocol accounts for differences in brain scaling, thus removing much of the effect of ICV, but we still included it as a covariate. This was run separately for the post-acute and chronic data. Results were corrected for multiple comparisons using searchlight FDR ${ }^{30}$ across the brain $(q<0.05)$. We tested the voxel-wise Jacobian maps for associations with our summary cognitive score with linear regression, covarying for age, sex, scanner, and ICV, both across all subjects (for which we also covaried for group) and within TBI only. We also tested the Jacobian maps for associations with GCS score upon hospital admission.

\section{Results}

\section{Post-acute}

In the post-acute phase, we found several areas of significant brain volume differences in the TBI group. The largest result was a volume expansion in the TBI group in the lateral ventricles, which had an average volume increase of $1.6 \%$ across the TBI group in this cluster. Ventricle expansion is indicative of atrophy of the nearby brain tissue, in this case the $\mathrm{CC}$ and subcortical structures such as the caudate and thalamic nuclei. We also found lower regional brain volumes in the TBI group in the left lingual gyrus, bilateral posterior cingulate gyrus, right fusiform gyrus, right superior temporal gyrus, left thalamus, left precuneus, left superior frontal gyrus, left orbital gyrus, as well as a large cluster corresponding to the right posterior cingulate gyrus, cingulum (hippocampal aspect), and parahippocampal gyrus. We also found an area of expansion in msTBI along the periphery around the pre- and post-central gyri. Increased volume in clusters on the periphery can indicate cerebrospinal fluid (CSF) expansion, suggesting cortical tissue loss. These results can be seen in Figure 1 and Table 2. ICV did not differ significantly between the groups. 


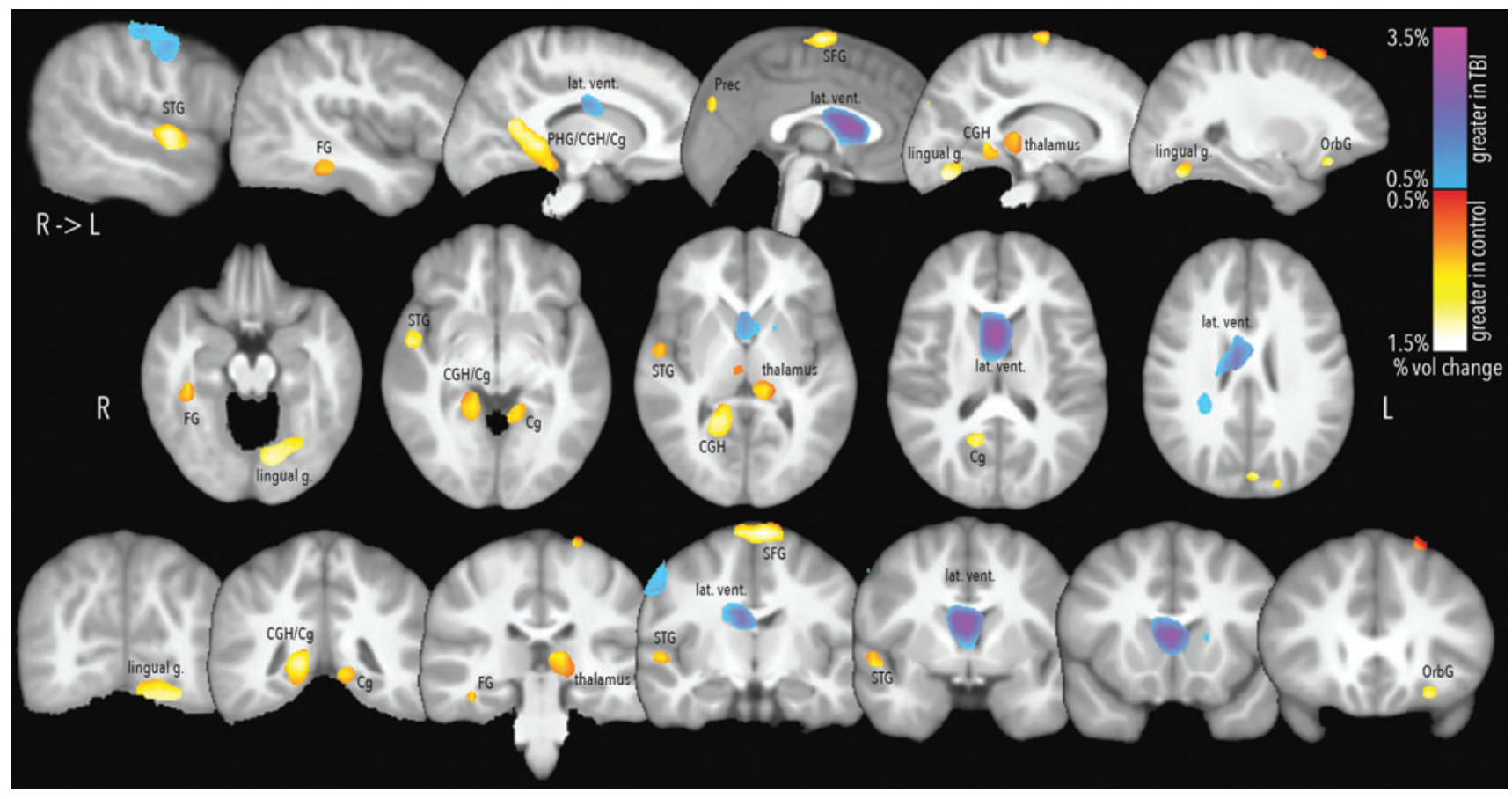

FIG. 1. Differences in regional brain volume in the post-acute phase between TBI and controls. Yellow-red areas are those whose area is greater in control than TBI, blue-purple areas are those whose area is greater in TBI. Percent volume change is shown in these color changes. Left in image is right in brain. $\mathrm{Cg}$, cingulum; CGH, cingulum (hippocampal aspect); lat. vent., lateral ventricles; OrbG, orbital gyrus; PCR, posterior corona radiata; PHG, parahippocampal gyrus; PoG, postcentralgyrus; Prec, precuneus; PrG, precentralgyrus; SFG, superior frontal gyrus; STG, superior temporal gyrus; TBI, traumatic brain injury. Color image is available online at www.liebertpub.com/neu

In the post-acute phase, we found several significant associations between regional volume in the msTBI group and GCS score. We found positive associations between GCS score and regional brain volume in the middle cerebellar peduncle, right middle and superior temporal gyri, right superior occipital gyral WM, and in an area that corresponds approximately to the cavernous sinus. We also found negative associations, where individuals with lower GCS score (more severe TBI) had greater volume, near the precuneus along the longitudinal fissure (perhaps representing the CSF space expansion in the falxcerebri and subarachnoid space), and near the left insula and left transverse temporal gyrus. These results can be seen in Fig. 2.

\section{Chronic}

In the chronic phase, we again found many areas of significantly lower volume in msTBI. As in the post-acute phase, we found ventricular expansion in TBI. The spatial extent of the cluster was smaller than the post-acute result, but the magnitude of volume reduction was greater: the average volume reduction across the lateral ventricle cluster was $1.6 \%$ in the post-acute phase and $2.0 \%$ in the chronic phase compared with the MDT. We found lower volume in msTBI compared with controls in the bilateral lingual gyrus, right middle temporal gyrus, bilateral orbital gyrus, right fusiform gyrus, anterior and mid-cingulate cortex, left superior parietal lobule, and left precentralgyrus. We found a few areas of apparent expansion in the msTBI group that were not easily explained by $\mathrm{CSF} /$ ventricular expansion. These included a small cluster in the left inferior frontal gyrus, and much larger clusters that overlapped with the posterior thalamic radiations (bilaterally), right superior longitudinal fasciculus, right occipital gyrus, right angular gyrus, and right superior parietal lobule. Interpreting these effects will require verification in future independent samples.
These results can be seen in Figure 3 and Table 2. ICV did not differ significantly between groups.

We did not find any significant associations between regional volume in the msTBI group and GCS score in the chronic phase.

\section{Relationships to cognitive performance}

In the post-acute phase, we found a number of associations between cognitive performance and regional brain volume across all participants and in a separate analysis restricted to the TBI group. Across all participants, better cognitive performance was associated with greater regional brain volumes in the bilateral inferior temporal gyrus, bilateral occipital gyrus, bilateral fusiform gyrus, and left superior temporal gyrus. Poorer cognitive performance was associated with greater volume in the lateral ventricles (indicating CSF expansion and tissue atrophy), the left occipital gyrus, left middle temporal gyrus, and the right cingulate gyrus. These results can be seen in Figure 4 and Table 3. In just the TBI participants, better cognitive performance was associated with greater brain volume in the bilateral superior frontal gyrus, bilateral fusiform gyrus, right occipital gyrus, right superior parietal lobule, right precuneus, right precentralgyrus, left inferior temporal gyrus, and a large area on the perimeter overlapping with the middle frontal gyrus (this may reflect an expansion of CSF rather than an expansion of tissue). Poorer cognitive performance was associated with greater volume in the lateral ventricles (indicating CSF expansion and tissue atrophy), the left occipital gyrus, and left transverse temporal gyrus. These results can be seen in Figure 5 and Table 3.

In the chronic phase, across all participants, better cognitive performance was associated with greater regional brain volumes in the bilateral post-central gyrus, bilateral insula, right middle cerebellar peduncle, left inferior temporal gyrus. Poorer cognitive 
TABle 2. Group Differences

\begin{tabular}{|c|c|c|c|c|c|c|c|}
\hline \multicolumn{8}{|c|}{ Post-acute time-point } \\
\hline \multirow[b]{2}{*}{ Cluster location } & \multirow[b]{2}{*}{ Size (voxel) } & \multirow[b]{2}{*}{ Peak \% change } & \multicolumn{3}{|c|}{ MNI coordinates (peak) } & \multirow[b]{2}{*}{ Side } & \multirow[b]{2}{*}{ Tissue } \\
\hline & & & $X$ & $Y$ & $Z$ & & \\
\hline \multicolumn{8}{|l|}{ Areas of atrophy in $T B I$} \\
\hline $\mathrm{PHG} / \mathrm{CGH} / \mathrm{Cg}$ & 6732 & $-1.30 \%$ & 16 & -42 & 2 & $\mathrm{R}$ & GM/WM \\
\hline Superior frontal gyrus & 3727 & $-1.40 \%$ & -6 & 0 & 72 & $\mathrm{~B}$ & GM \\
\hline Lingual gyrus & 2887 & $-1.30 \%$ & -12 & -78 & -14 & $\mathrm{~L}$ & GM \\
\hline Superior temporal gyrus & 1858 & $-1.30 \%$ & 54 & -4 & -4 & $\mathrm{R}$ & GM/WM \\
\hline Thalamus & 1720 & $-1.00 \%$ & -12 & -30 & 6 & $\mathrm{~L}$ & GM \\
\hline Cingulate gyrus (posterior) & 897 & $-0.90 \%$ & -14 & -48 & 2 & $\mathrm{~L}$ & GM \\
\hline Fusiform gyrus & 732 & $-0.88 \%$ & 40 & -34 & -18 & $\mathrm{R}$ & GM \\
\hline Precuneus & 488 & $-1.20 \%$ & -4 & -78 & 36 & $\mathrm{~L}$ & GM \\
\hline Orbital gyrus (posterior) & 227 & $-1.10 \%$ & -26 & 26 & -20 & $\mathrm{~L}$ & GM \\
\hline \multicolumn{8}{|c|}{ Areas of volume expansion in TBI } \\
\hline Lateral ventricle & 12694 & $3.40 \%$ & 0 & 10 & 10 & $\mathrm{~B}$ & CSF \\
\hline Precentral/post-central gyrus & 3325 & $1.10 \%$ & 56 & 2 & 44 & $\mathrm{R}$ & $\mathrm{GM} / \mathrm{CSF}$ \\
\hline Posterior corona radiata & 757 & $0.82 \%$ & 28 & -34 & 26 & $\mathrm{R}$ & WM \\
\hline \multicolumn{8}{|c|}{ Chronic time-point } \\
\hline & & & \multicolumn{3}{|c|}{ MNI coordinates (peak) } & & \\
\hline Cluster location & Size (voxel) & Peak \% change & $X$ & $Y$ & $Z$ & Side & Tissue \\
\hline \multicolumn{8}{|l|}{ Areas of atrophy in $T B I$} \\
\hline Lingual gyrus & 11283 & $-2.5 \%$ & -32 & -72 & -20 & $\mathrm{~B}$ & GM/WM \\
\hline Occipital gyrus & 3047 & $-1.2 \%$ & 16 & -82 & 42 & $\mathrm{R}$ & GM \\
\hline Cingulate gyrus (mid) & 1237 & $-1.7 \%$ & 8 & -24 & 42 & $\mathrm{R}$ & GM \\
\hline Middle temporal gyrus & 1121 & $-1.3 \%$ & 48 & 14 & -28 & $\mathrm{R}$ & GM \\
\hline Orbital gyrus (medial) & 637 & $-0.96 \%$ & -12 & 34 & -26 & $\mathrm{~L}$ & GM \\
\hline Superior parietal lobule & 557 & $-1.8 \%$ & -20 & -68 & 46 & $\bar{L}$ & GM \\
\hline Orbital gyrus (medial) & 556 & $-1.0 \%$ & 10 & 34 & -26 & $\mathrm{R}$ & GM \\
\hline Precentralgyrus & 504 & $-1.6 \%$ & -22 & -16 & 66 & $\mathrm{~L}$ & GM \\
\hline Cingulate gyrus (anterior) & 215 & $-1.3 \%$ & 10 & 42 & 8 & $\mathrm{R}$ & GM \\
\hline Superior frontal gyrus & 208 & $-1.3 \%$ & 24 & 56 & 12 & $\mathrm{R}$ & GM \\
\hline \multicolumn{8}{|c|}{ Areas of volume expansion in $T B I$} \\
\hline SLF/PTR/AngG/SPL/Og & 9893 & $1.6 \%$ & 34 & -60 & 18 & $\mathrm{R}$ & WM \\
\hline Lateral ventricle & 6726 & $4.1 \%$ & 2 & 10 & 10 & $\mathrm{~B}$ & CSF \\
\hline Posterior thalamic radiation & 3971 & $2.0 \%$ & -26 & -74 & 14 & $\mathrm{~L}$ & WM \\
\hline Inferior frontal gyrus & 1739 & $1.7 \%$ & 48 & 32 & 14 & $\mathrm{R}$ & GM \\
\hline Inferior frontal gyrus & 917 & $1.6 \%$ & -40 & 48 & -4 & $\mathrm{~L}$ & GM \\
\hline
\end{tabular}

Group differences in regional brain volume in the post-acute and chronic time-points. For each cluster, the location, size, peak percent change, MNI coordinates of the peak, laterality, and the tissue type.

AngG, angular gyrus; $\mathrm{CG}$, cingulum; CGH, hippocampal part of the cingulum; CSF, cerebrospinal fluid; GM, gray matter; MNI, Montreal Neurological Institute; Og, occipital gyrus; PHG, parahippocampal gyrus; PTR, posterior thalamic radiation; SLF, superior longitudinal fasciculus; SPL, superior parietal lobule; TBI, traumatic brain injury.

performance was associated with greater volume in the lateral ventricles (indicating CSF expansion and tissue atrophy), the left entorhinal cortex, and an area overlapping with the left superior temporal gyrus and inferior frontal gyrus on the periphery, likely indicating CSF expansion and tissue loss. These results can be seen in Figure 6 and Table 4. In just the TBI participants, better cognitive performance was associated with greater brain volume in the right middle cerebellar peduncle, right orbital gyrus, and bilateral fusiform gyrus. Poorer cognitive performance was associated with greater volume in lateral ventricles (indicating CSF expansion and tissue atrophy), bilateral middle frontal gyrus, right hippocampus, right middle/superior temporal gyrus, left amygdala, left fornix, left inferior temporal gyrus, left supramarginal gyrus, and an area overlapping with the left superior temporal gyrus and inferior frontal gyrus on the periphery, likely indicating CSF expansion and tissue loss. These results can be seen in Figure 7 and Table 4 .

\section{Discussion}

This study revealed patterns of volume differences in pediatric msTBI patients. Many of these effects were detected at two distinct time-points post-injury. Although TBI is a heterogeneous disorder, our results indicate that there are some common areas of disruption within the TBI group. In the post-acute phase, we found several areas of reduced volume relative to the controls, as well as several areas of volume increase mostly explained by CSF space expansion. Similarly in the chronic phase, we found several of these effects persisting, even becoming more pronounced. Planned 


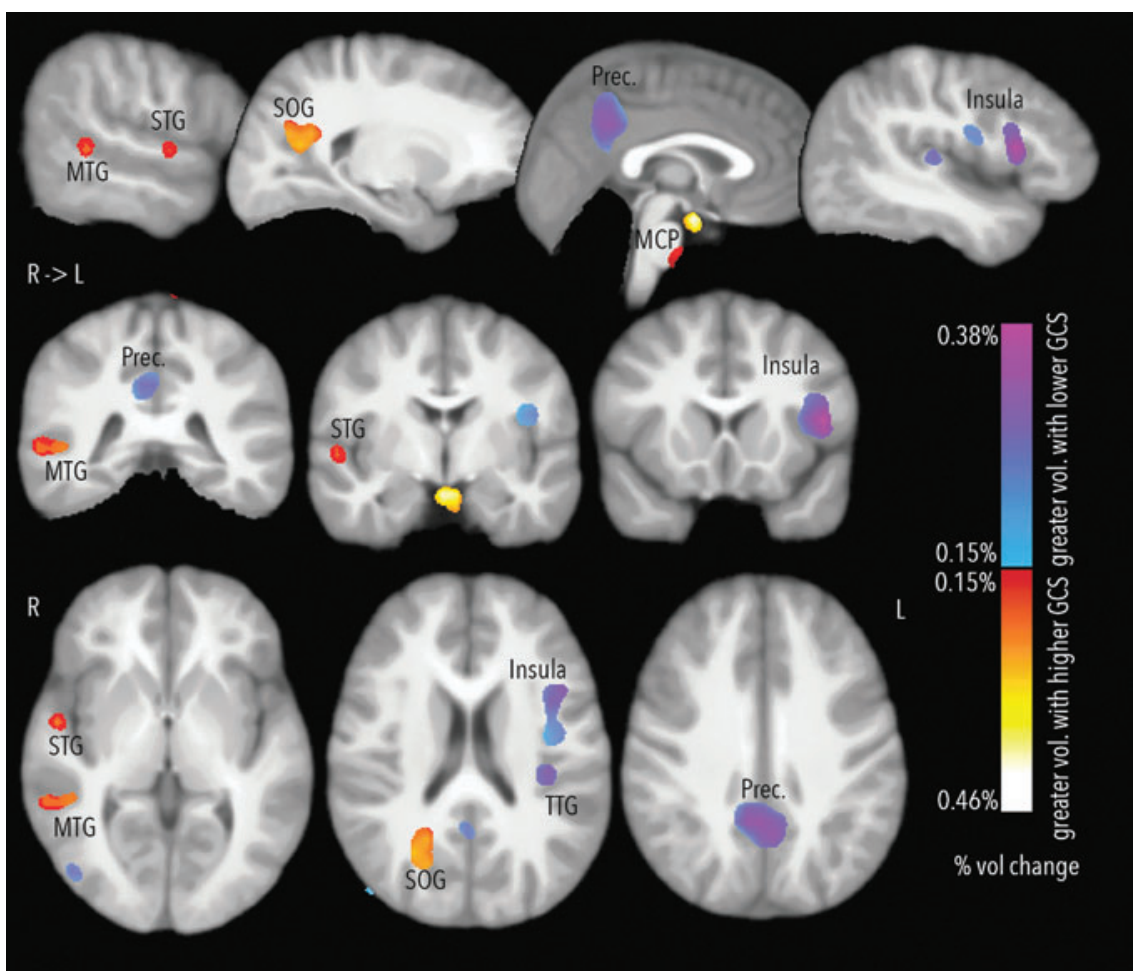

FIG. 2. Correlations between regional brain volume and GCS score, within the post-acute TBI group. Yellow-red areas are those whose area is greater with higher GCS score (less severe), blue-purple areas are those whose area is greater with lower GCS score (more severe). Percent volume change is shown in these color changes. Left in image is right in brain. GCS, Glasgow Coma Scale; Prec, precuneus; MCP, middle cerebellar peduncle; MTG, middle temporal gyrus; SOG, superior occipital gyrus; STG, superior temporal gyrus; TTG, transverse temporal gyrus. Color image is available online at www.liebertpub.com/neu

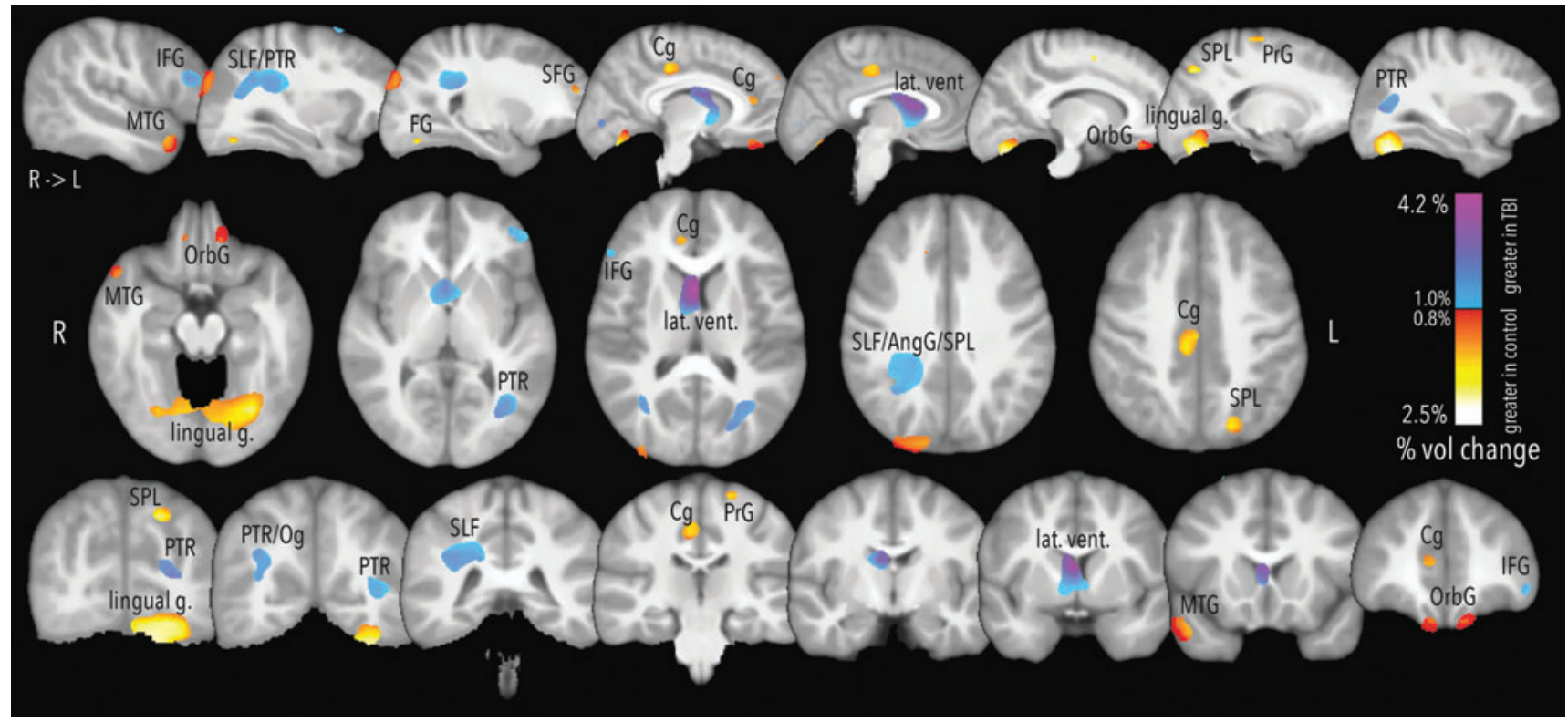

FIG. 3. Differences in regional brain volume in the chronic phase between TBI and controls. Yellow-red areas are those whose area is greater in control than TBI, blue-purple areas are those whose area is greater in TBI. Percent volume change is shown in these color changes. Left in image is right in brain. AngG, angular gyrus; Cg, cingulum; FG, fusiform gyrus; IFG, inferior frontal gyrus; lat. vent., lateral ventricles; MTG, middle temporal gyrus; OrbG, orbital gyrus; PrG, precentralgyrus; PTR, posterior thalamic radiation; SFG, superior frontal gyrus; SLF, superior longitudinal fasciculus; SPL, superior parietal lobule; TBI, traumatic brain injury. Color image is available online at www.liebertpub.com/neu 


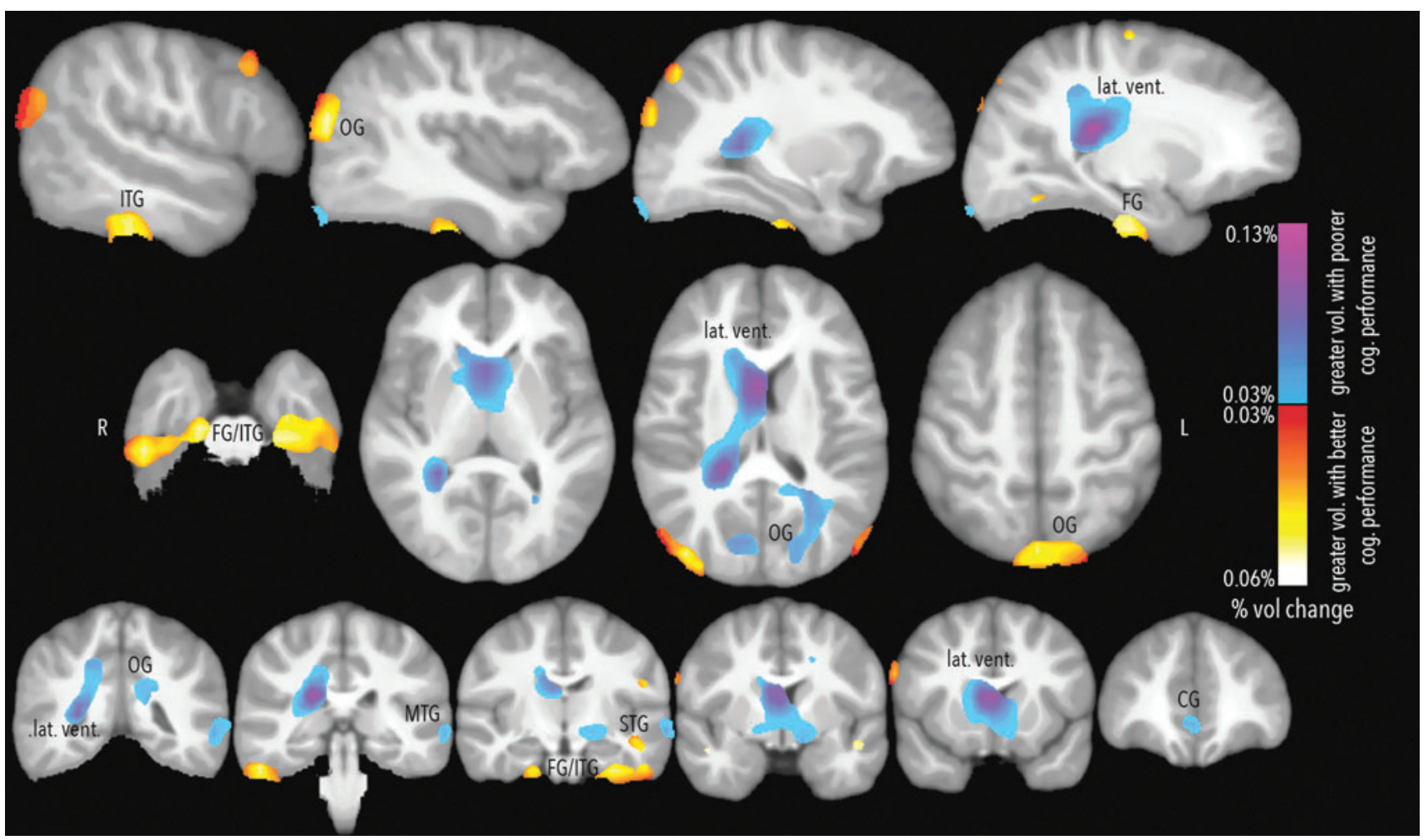

FIG. 4. Regional associations with cognitive performance across all participants in the post-acute phase. Yellow-red areas are those where greater volume was associated with better cognitive performance, blue-purple areas are those where greater volume was associated with poorer cognitive performance. Percent volume change is shown in these color changes. Left in image is right in brain. FG, fusiform gyrus; ITG, inferior temporal gyrus; lat. vent., lateral ventricles; OG, occipital gyrus; MTG, middle temporal gyrus; STG, superior temporal gyrus. Color image is available online at www.liebertpub.com/neu

longitudinal analyses will reveal whether these effects are truly progressive over this period within subjects. We were also able to link some of these volumetric differences to deficits in cognitive performance, suggesting that regional brain volume could be a useful biomarker of recovery and a target of interventions.

In the post-acute and chronic phases we found significant ventricular expansion, suggesting tissue loss in the surrounding CC and subcortical structures. This is consistent with prior studies of regional brain volume in TBI in adults ${ }^{7,9,12,13}$ and children. ${ }^{17,18} \mathrm{Al}-$ though only a true longitudinal assessment will show the progression of this tissue expansion, between the post-acute and chronic time-points, the spatial extent of the expansion appeared smaller in the chronic phase, whereas the magnitude of the change appeared greater. Increasing atrophy from the post-acute phase to the chronic phase in the tissues surrounding the lateral ventricles is supported by prior longitudinal studies in adults ${ }^{4,6}$ and children ${ }^{15,18}$ showing expansion of the lateral ventricles and atrophy in the CC and subcortical structures. In the current study the average volume increase across the lateral ventricle cluster in the post-acute TBI group relative to controls was $1.6 \%$, whereas in the chronic phase it was $2.0 \%$. For comparison, ventricular expansion in MCI (mild cognitive impairment, the prodromal phase of Alzheimer's disease) is $10-15 \%$ for MCI and over $20 \%$ for Alzheimer's, ${ }^{26}$ but it should be noted that older individuals have years to accumulate these changes. Volume loss in the left lingual gyrus was also seen across both time-points, with the chronic TBI individuals also showing volume deficits in the right lingual gyrus. In the chronic phase, this cluster was both larger in spatial extent and magnitude than in the post-acute phase (1\% volume change compared with controls in the post-acute phase versus $1.5 \%$ in the chronic phase). We found lower volume in clusters in the right fusiform gyrus across both time-points, although they did not overlap spatially. We similarly found clusters in the orbital gyrus (bilateral in chronic, just left in post-acute) across both time-points, non-overlapping. Lastly, in the post-acute phase there was a large cluster of reduced volume in an area overlapping the bilateral posterior cingulate, parahippocampal gyrus, and the hippocampal part of the cingulum (CGH). In the chronic phase, we found significant reductions in volume of the mid and anterior cingulate cortex. Volume losses in the anterior, mid, and posterior cingulate have been reported by a number of prior studies, ${ }^{5-8}$ as has volume loss in the parahippocampal cortex ${ }^{6}$ and hippocampus. ${ }^{10,11}$

Turning to significant differences only detected in the post-acute phase, we found a large cluster in the left posterior thalamus, likely the pulvinar. The average volume difference compared with controls across this cluster was $0.7 \%$. Several prior studies have reported reduced volume in the thalamus, and pulvinar specifically, including several studies that assessed volume loss in TBI patients longitudinally. ${ }^{4,6,7,12} \mathrm{We}$ also found clusters of lower volume in TBI in the superior frontal gyrus (bilaterally) and right superior temporal gyrus, which have been reported before in both children and adults. ${ }^{6-8,16}$ When examining associations with injury severity (GCS), we found a few clusters of lower volume in more severely injured individuals, but we also found the opposite-areas of greater volume in more severely injured participants. Rather than interpreting these results as truly representing tissue expansion in 
Table 3. Post-acute Association with Cognitive Summary Index

\begin{tabular}{|c|c|c|c|c|c|c|c|}
\hline \multicolumn{8}{|c|}{ All participants } \\
\hline \multirow[b]{2}{*}{ Cluster location } & \multirow[b]{2}{*}{ Size (voxel) } & \multirow[b]{2}{*}{ Peak \% change } & \multicolumn{3}{|c|}{ MNI coordinates (peak) } & \multirow[b]{2}{*}{ Side } & \multirow[b]{2}{*}{ Tissue } \\
\hline & & & $X$ & $Y$ & $Z$ & & \\
\hline \multicolumn{8}{|c|}{ Greater volume associated with better cognitive performance } \\
\hline STG/MTG/ITG/FG & 7999 & $0.06 \%$ & -46 & -2 & -18 & $\mathrm{~L}$ & GM/WM \\
\hline ITG/FG & 6208 & $0.05 \%$ & 22 & -24 & -32 & $\mathrm{R}$ & GM \\
\hline Occipital gyrus & 6046 & $0.05 \%$ & 42 & -78 & 28 & $\mathrm{R}$ & GM \\
\hline Precuneus & 4884 & $0.05 \%$ & 0 & -70 & 60 & $\mathrm{~B}$ & GM \\
\hline Pre-central gyrus & 1988 & $0.03 \%$ & 62 & 10 & 24 & $\mathrm{R}$ & $\mathrm{GM} / \mathrm{CSF}$ \\
\hline Precuneus & 1250 & $0.03 \%$ & -52 & -74 & 26 & $\mathrm{~L}$ & GM/CSF \\
\hline Angular gyrus & 738 & $0.05 \%$ & 28 & -70 & 54 & $\mathrm{R}$ & GM \\
\hline Superior frontal gyrus & 429 & $0.05 \%$ & 16 & -10 & 68 & $\mathrm{R}$ & GM \\
\hline Orbital gyrus (straight) & 237 & $0.03 \%$ & 0 & 26 & -30 & $\mathrm{~L}$ & GM \\
\hline \multicolumn{8}{|c|}{ Greater volume associated with poorer cognitive performance } \\
\hline Lateral ventricles & 45327 & $0.13 \%$ & 2 & 10 & 10 & $\mathrm{~B}$ & $\mathrm{CSF}$ \\
\hline Lat. vent./PTR & 11378 & $0.06 \%$ & -26 & -54 & 18 & $\mathrm{~L}$ & $\mathrm{CSF} / \mathrm{WM}$ \\
\hline MTG/STG & 5506 & $0.05 \%$ & -66 & -18 & -6 & $\mathrm{~L}$ & GM \\
\hline Cuneus & 1757 & $0.06 \%$ & 8 & -74 & 28 & $\mathrm{R}$ & GM \\
\hline Inferior frontal gyrus & 773 & $0.05 \%$ & -46 & 28 & -12 & $\mathrm{~L}$ & GM \\
\hline Cingulate gyrus (anterior) & 689 & $0.04 \%$ & 2 & 36 & -8 & $\mathrm{R}$ & GM \\
\hline
\end{tabular}

Just TBI participants

\begin{tabular}{|c|c|c|c|c|c|c|c|}
\hline \multirow[b]{2}{*}{ Cluster location } & \multirow[b]{2}{*}{ Size (voxel) } & \multirow[b]{2}{*}{ Peak \% change } & \multicolumn{3}{|c|}{ MNI coordinates (peak) } & \multirow[b]{2}{*}{ Side } & \multirow[b]{2}{*}{ Tissue } \\
\hline & & & $X$ & $Y$ & $Z$ & & \\
\hline \multicolumn{8}{|c|}{ Greater volume associated with better cognitive performance } \\
\hline Occipital gyrus & 13039 & $0.08 \%$ & 40 & -78 & 28 & $\mathrm{R}$ & GM \\
\hline Occipital/fusiform gyrus & 8030 & $0.07 \%$ & 22 & -72 & -18 & $\mathrm{R}$ & GM \\
\hline Middle frontal gyrus & 7916 & $0.06 \%$ & 54 & 28 & 28 & $\mathrm{R}$ & GM \\
\hline Fusiform gyrus & 5025 & $0.05 \%$ & -24 & -58 & -16 & $\mathrm{~L}$ & GM \\
\hline Occipital gyrus & 4520 & $0.05 \%$ & -52 & -72 & 26 & $\bar{L}$ & GM \\
\hline $\begin{array}{l}\text { Precuneus } \\
\text { Prestas }\end{array}$ & 3772 & $0.09 \%$ & 14 & -56 & 60 & $\mathrm{R}$ & GM \\
\hline Superior frontal gyrus & 1661 & $0.06 \%$ & -6 & 58 & 22 & $\mathrm{~L}$ & GM \\
\hline Superior frontal gyrus & 1165 & $0.06 \%$ & 22 & 64 & 12 & $\mathrm{R}$ & GM \\
\hline Fusiform gyrus & 1086 & $0.05 \%$ & -46 & -32 & -26 & $\mathrm{~L}$ & GM \\
\hline Post-central gyrus & 638 & $0.06 \%$ & 56 & -18 & 42 & $\mathrm{R}$ & GM \\
\hline Cuneus & 592 & $0.05 \%$ & 2 & -92 & 30 & $\mathrm{~B}$ & GM/CSF \\
\hline Inferior temporal gyrus & 285 & $0.09 \%$ & -40 & 0 & -32 & $\mathrm{~L}$ & GM/WM \\
\hline Superior parietal lobule & 216 & $0.06 \%$ & 36 & -42 & 54 & $\mathrm{R}$ & GM \\
\hline Precentralgyrus & 115 & $0.05 \%$ & 14 & -20 & 72 & $\mathrm{R}$ & GM \\
\hline \multicolumn{8}{|c|}{ Greater volume associated with poorer cognitive performance } \\
\hline Lateral ventricle & 32175 & $0.17 \%$ & 2 & 10 & 10 & $\mathrm{~B}$ & $\mathrm{CSF}$ \\
\hline Occipital gyrus & 2967 & $0.06 \%$ & -28 & -58 & 20 & $\mathrm{~L}$ & WM \\
\hline Transverse temporal gyrus & 420 & $0.04 \%$ & -34 & -24 & 12 & $\mathrm{~L}$ & GM \\
\hline
\end{tabular}

Correlations between the cognitive performance score and regional brain volume across all post-acute participants (upper portion) and just in the TBI participants (lower portion). For each cluster, the location, size, peak percent change, MNI coordinates of the peak, laterality, and the tissue type.

CSF, cerebrospinal fluid; FG, fusiform; GM, gray matter; ITG, inferior temporal gyrus; MNI, Montreal Neurological Institute; MTG, middle temporal gyrus; PTR, posterior thalamic radiation; STG, superior temporal gyrus; TBI, traumatic brain injury; WM, white matter.

the more severely injured participants, we think it is more likely that our results indicate expansion due to injury-related processes, such as inflammation. An earlier examination of this dataset including metrics from MRS (magnetic resonance spectroscopy), which measures brain metabolites that can indicate demyelination, inflammation, or neuronal loss, found inflammatory markers in the post-acute phase but no longer in the chronic phase. ${ }^{31}$ Prior research suggests that whereas edema peaks $24-72 \mathrm{~h}$ post-injury, ${ }^{32}$ inflammation caused by TBI is a more long-term problem, causing continued WM disruption. ${ }^{33,}{ }^{34}$ In particular, the cluster spanning the midline in the precuneus may be detecting injury effects leading to expanded CSF space between the hemispheres.

In the chronic phase, we detected some group differences not seen in the post-acute phase. There were several small clusters of reduced volume in msTBI, including the middle temporal gyrus, superior frontal gyrus (far more anterior than the cluster in the postacute phase), superior parietal lobule, and pre-central gyrus. Reductions in frontal, temporal, and parietal cortices generally have been found in prior studies. ${ }^{6-8,16} \mathrm{We}$ also found several additional clusters of volume expansion in the TBI cohort, not easily 


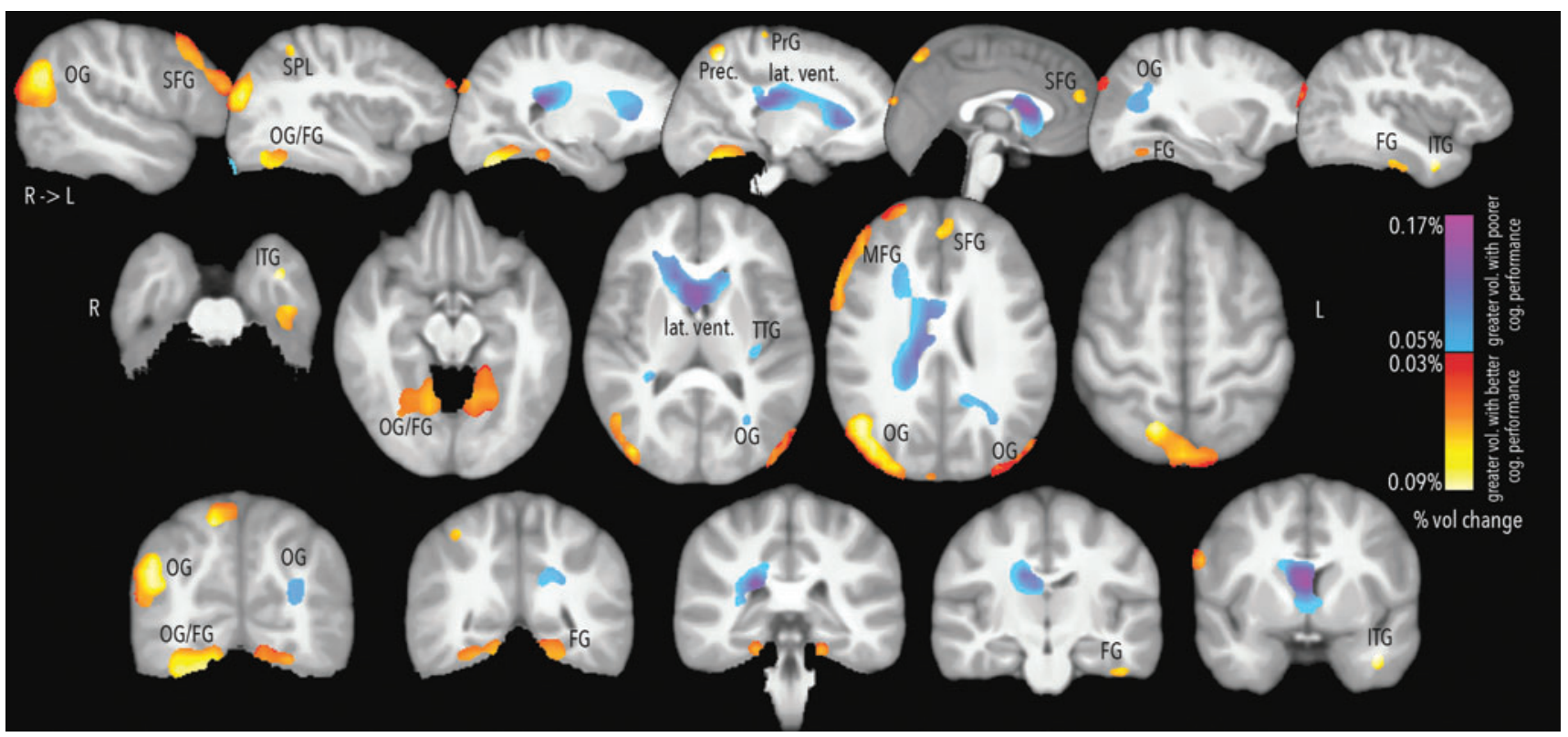

FIG. 5. Regional associations with cognitive performance across just TBI participants in the post-acute phase. Yellow-red areas are those where greater volume was associated with better cognitive performance, blue-purple areas are those where greater volume was associated with poorer cognitive performance. Percent volume change is shown in these color changes. Left in image is right in brain. FG, fusiform gyrus; ITG, inferior temporal gyrus; lat. vent., lateral ventricles; MFG, middle frontal gyrus; OG, occipital gyrus; Prec., precuneus; PrG, precentralgyrus; SFG, superior frontal gyrus; SPL, superior parietal lobule; TBI, traumatic brain injury; TTG, transverse temporal gyrus. Color image is available online at www.liebertpub.com/neu

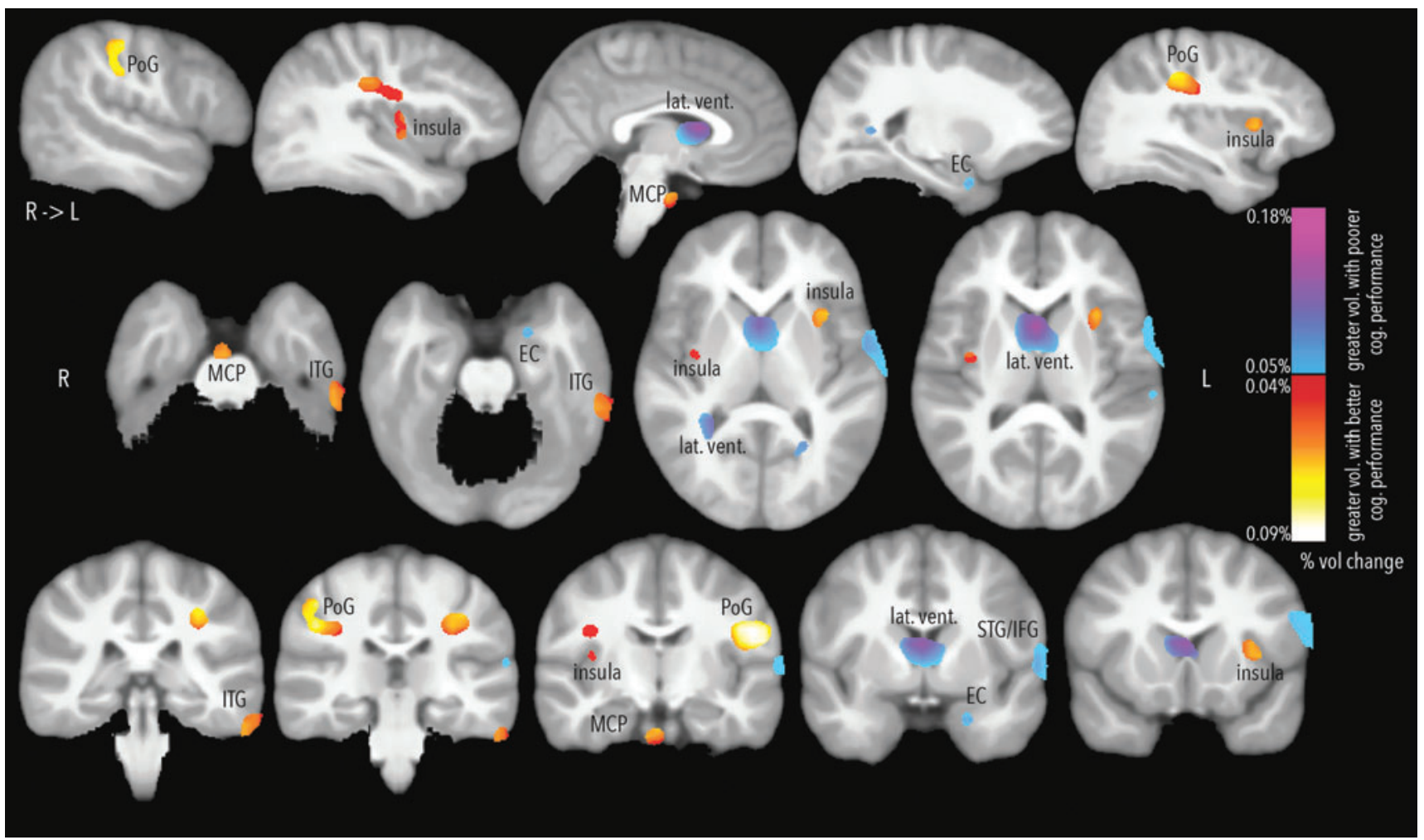

FIG. 6. Regional associations with cognitive performance across all participants in the chronic phase. Yellow-red areas are those where greater volume was associated with better cognitive performance, blue-purple areas are those where greater volume was associated with poorer cognitive performance. Percent volume change is shown in these color changes. Left in image is right in brain. EC, entorhinal cortex; IFG, inferior frontal gyrus; ITG, inferior temporal gyrus; lat. vent., lateral ventricles; MCP, middle cerebellar peduncle; PoG, postcentralgyrus; STG, superior temporal gyrus. Color image is available online at www.liebertpub.com/neu 
Table 4. Chronic Association with Cognitive Summary Index

\begin{tabular}{|c|c|c|c|c|c|c|c|}
\hline \multicolumn{8}{|c|}{ All participants } \\
\hline \multirow[b]{2}{*}{ Cluster location } & \multirow[b]{2}{*}{ Size (voxel) } & \multirow[b]{2}{*}{ Peak \% change } & \multicolumn{3}{|c|}{ MNI coordinates (peak) } & \multirow[b]{2}{*}{ Side } & \multirow[b]{2}{*}{ Tissue } \\
\hline & & & $X$ & $Y$ & $Z$ & & \\
\hline \multicolumn{8}{|c|}{ Greater volume associated with better cognitive performance } \\
\hline Post-central gyrus & 6978 & $0.09 \%$ & -52 & -8 & 24 & $\mathrm{~L}$ & GM \\
\hline Post-central gyrus & 3185 & $0.07 \%$ & 48 & -22 & 46 & $\mathrm{R}$ & GM/WM \\
\hline Inferior temporal gyrus & 1713 & $0.04 \%$ & -60 & -40 & -26 & $\mathrm{~L}$ & GM \\
\hline Insula & 706 & $0.05 \%$ & -36 & 16 & 2 & $\mathrm{~L}$ & GM \\
\hline Middle cerebellar peduncle & 555 & $0.06 \%$ & 6 & -14 & -34 & $\mathrm{R}$ & WM \\
\hline Insula & 471 & $0.04 \%$ & 38 & -6 & 0 & $\mathrm{R}$ & GM \\
\hline \multicolumn{8}{|c|}{ Greater volume associated with poorer cognitive performance } \\
\hline Lateral ventricle & 4987 & $0.18 \%$ & 0 & 10 & 10 & $\mathrm{~B}$ & CSF \\
\hline STG/IFG (CSF expansion) & 4545 & $0.07 \%$ & -62 & 2 & 4 & $\mathrm{~L}$ & $\mathrm{CSF}$ \\
\hline Lateral ventricle & 764 & $1.00 \%$ & 28 & -44 & 10 & $\mathrm{R}$ & $\mathrm{CSF}$ \\
\hline Entorhinal cortex & 175 & $0.06 \%$ & -22 & 0 & -30 & $\mathrm{~L}$ & GM \\
\hline Lateral ventricle & 150 & $0.07 \%$ & -24 & -56 & 12 & $\mathrm{~L}$ & $\mathrm{CSF} / \mathrm{WM}$ \\
\hline
\end{tabular}

\begin{tabular}{|c|c|c|c|c|c|c|c|}
\hline \multicolumn{8}{|c|}{ Just TBI participants } \\
\hline \multirow[b]{2}{*}{ Cluster location } & \multirow[b]{2}{*}{ Size (voxel) } & \multirow[b]{2}{*}{ Peak \% change } & \multicolumn{3}{|c|}{ MNI coordinates (peak) } & \multirow[b]{2}{*}{ Side } & \multirow[b]{2}{*}{ Tissue } \\
\hline & & & $X$ & $Y$ & $Z$ & & \\
\hline \multicolumn{8}{|c|}{ Greater volume associated with better cognitive performance } \\
\hline Orbital gyrus (medial/posterior) & 1014 & $0.08 \%$ & 20 & 20 & -26 & $\mathrm{R}$ & GM \\
\hline Middle cerebellar peduncle & 760 & $0.07 \%$ & 0 & -12 & -26 & $\mathrm{R}$ & WM \\
\hline Fusiform gyrus & 382 & $0.08 \%$ & -24 & -34 & -26 & $\mathrm{~L}$ & GM \\
\hline Fusiform gyrus & 272 & $0.07 \%$ & 22 & -30 & -26 & $\mathrm{R}$ & GM \\
\hline \multicolumn{8}{|c|}{ Greater volume associated with poorer cognitive performance } \\
\hline Lateral ventricle & 16027 & $0.32 \%$ & 0 & 12 & 10 & $\mathrm{~B}$ & CSF \\
\hline Inferior temporal gyrus & 2955 & $0.11 \%$ & -46 & -44 & -10 & $\mathrm{~L}$ & GM/WM \\
\hline STG/IFG (CSF expansion) & 1998 & $0.09 \%$ & -60 & -2 & 4 & $\mathrm{~L}$ & CSF \\
\hline Middle frontal gyrus & 1521 & $0.09 \%$ & 34 & 48 & -4 & $\mathrm{R}$ & GM \\
\hline Hippocampus & 731 & $0.07 \%$ & 28 & -6 & -22 & $\mathrm{R}$ & GM \\
\hline Supramarginal gyrus & 639 & $0.10 \%$ & -56 & -28 & 18 & $\mathrm{~L}$ & GM \\
\hline Amygdala & 624 & $0.10 \%$ & -20 & 0 & -30 & $\mathrm{~L}$ & GM \\
\hline Middle frontal gyrus & 583 & $0.10 \%$ & -46 & 18 & 24 & $\mathrm{~L}$ & GM \\
\hline Fornix & 310 & $0.05 \%$ & -32 & -36 & 2 & $\mathrm{~L}$ & WM \\
\hline Middle/superior temporal gyrus & 151 & $0.05 \%$ & 56 & 8 & -20 & $\mathrm{R}$ & GM \\
\hline
\end{tabular}

Correlations between the cognitive performance score and regional brain volume across all chronic participants (upper portion) and just in the TBI participants (lower portion). For each cluster, the location, size, peak percent change, MNI coordinates of the peak, laterality, and the tissue type.

CSF, cerebrospinal fluid; IFG, inferior frontal gyrus; GM, gray matter; MNI, Montreal Neurological Institute; STG, superior temporal gyrus; TBI, traumatic brain injury; WM, white matter.

explainable by CSF expansion. A very large WM cluster in the right hemisphere was detected overlapping multiple regions (occipital gyral WM, angular gyral WM, superior parietal lobule WM, superior longitudinal fasciculus, and posterior thalamic radiations), as well as a smaller associated cluster in the left hemisphere. This may be evidence of recovery, but there are other plausible explanations as well. Kim and colleagues ${ }^{7}$ suggested that structural atrophy could lead to reduced compression of axonal tracts, which could be picked up by our methods as an apparent expansion. Reduced compression of tracts would be associated with decreased WM integrity of those tracts, as reflected in reduced FA (fractional anisotropy-the degree to which water diffuses in one direction, along well-myelinated axons), increased MD (mean diffusivity) and increased RD (radial diffusivity). This is precisely what we found in a recent tract-based examination of the WM integrity of these participants. ${ }^{21} \mathrm{We}$ found higher MD and RD in TBI partici- pants in the parietal projections of a number of tract systems overlapping with these results. This could be contributing to the volume expansion we see here.

Our examination of cognitive performance yielded widespread significant associations with regional brain volume, across both time-points, across all subjects and upon follow-up in just the TBI group. This is the first investigation of the performance index for association with neuroimaging markers. The results presented here indicate that the cognitive summary index is a sensitive marker of the neurological deficits of TBI. The cognitive summary index includes tests that tap cognitive domains separately, including processing speed, visuospatial function, working memory, attention, and executive functions examined in prior studies of children with TBI. In prior studies of children with TBI, processing speed and visuospatial function were positively correlated with $\mathrm{CC}$ area, and moderately related to ventricular enlargement. ${ }^{17}$ In adults, 


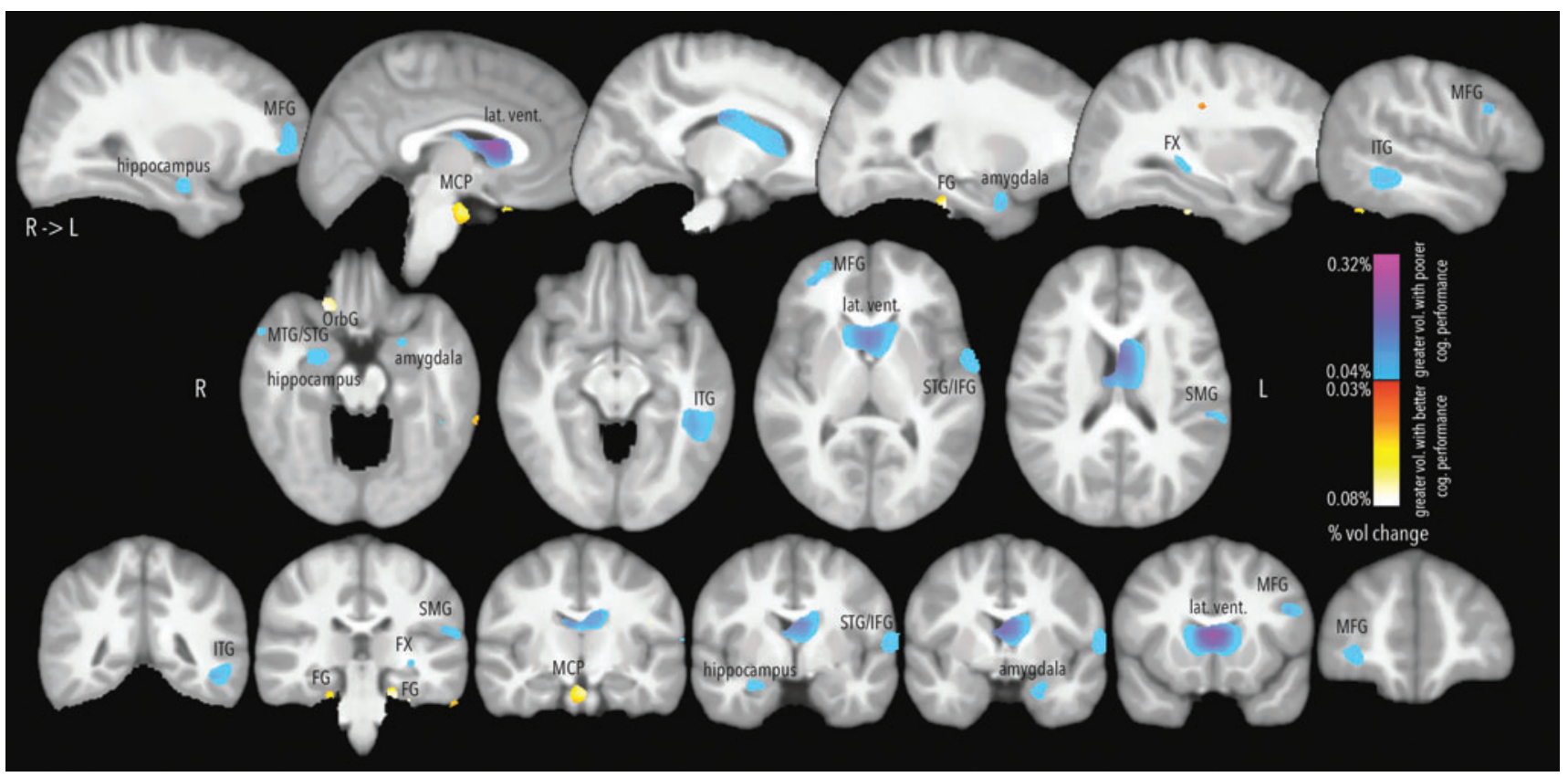

FIG. 7. Regional associations with cognitive performance across just TBI participants in the chronic phase. Yellow-red areas are those where greater volume was associated with better cognitive performance, blue-purple areas are those where greater volume was associated with poorer cognitive performance. Percent volume change is shown in these color changes. Left in image is right in brain. FG, fusiform; FX, fornix; IFG, inferior frontal gyrus; ITG, inferior temporal gyrus; lat. vent., lateral ventricles; MCP, middle cerebellar peduncle; MFG, middle frontal gyrus; OrbG, orbital gyrus; SMG, supramarginal gyrus; STG, superior temporal gyrus; TBI, traumatic brain injury. Color image is available online at www.liebertpub.com/neu

deficits in processing speed were associated with volume loss in the brainstem and cerebellum. ${ }^{6}$ Deficits in memory and executive function were associated with volume loss in the anterior cingulate, hippocampus, and lateral ventricle expansion. ${ }^{6,13}$ The main finding that was consistent across both time-points, whether all subjects were included (with group as a covariate) or just the TBI group, was that poorer cognitive performance was associated with expansion of the lateral ventricles. This indicates that the cognitive performance measure is sensitive to variation even within individuals with TBI, which could prove useful in tracking their neurocognitive recovery. Many more areas of atrophy were associated with poorer cognitive function in the post-acute phase than in the chronic phase. This most likely is due to the reduced statistical power resulting from the smaller number of participants in the chronic phase. This finding merits a true longitudinal follow-up. However, it could also indicate cognitive or neural recovery. Whereas the average cognitive summary index score in the TBI group decreased from the post-acute to chronic time-points, more than half of the TBI patients improved in their cognitive performance over this period. This raises a key issue in TBI: heterogeneity in outcome and how to make better predictions. Future longitudinal studies will examine how regional volume and cognitive performance change over the first year post-injury, and whether they track together.

A major focus of future longitudinal studies will be to use both MRS and TBM to test the hypothesis that post-acute inflammation predicts the extent of continued tissue loss through the chronic phase. The results of this study suggest that such studies might focus on ventricular expansion as the outcome during the chronic phase. Additionally, future longitudinal studies, by examining the association between the rate and location of changes with measures of cognitive recovery, will help clarify the role that these changes play. Would slowing or halting brain atrophy, if possible, be beneficial as it would save tissue and speed recovery? Or alternatively, is atrophy a necessary part of recovery, as damaged tissue is cleared away to make room for reconnection and recovery? This is a complex question, which will need to be approached from multiple levels, but longitudinal imaging of tissue changes and tracking cognitive function will be key.

The heterogeneity of TBI is a consideration in brain imaging studies. Patients with msTBI have heterogeneity in injury type and severity, which can make population studies difficult. Prior to the non-linear registration that generated the Jacobian maps, we linearly register the images to the template, checking them visually for registration quality. In the case of an individual with localized damage that could potentially impact linear registration quality, ensuring good registration is critical for the next step, to minimize the impact of the local damage. Localized regions of damage will differ between TBI patients, but the methods we used here identified regions of reduced volume that were common across patients, and perhaps persist over the first year post-injury. Unlike prior studies of pediatric TBI patients, we did not find significant differences in $\mathrm{ICV},{ }^{10}$ this is likely because of our methods- 9 DOF linear registration to some degree adjusts for ICV, thus removing this potential effect. Tasker and associates ${ }^{10}$ further examined differences within TBI patients, showing an effect of length of hospitalization.

Here we present cross-sectional data revealing some consistent areas of brain atrophy among pediatric TBI patients, and several of these regions of atrophy are present across the first year post-injury. We further linked these volume deficits to deficits in cognitive performance, indicating that regional brain volume is a good candidate biomarker for impaired function in TBI. Future longitudinal studies 
using these data will shed light on the processes underlying recovery, and they should further our understanding of heterogeneity in postinjury outcome.

\section{Acknowledgments}

This study was supported by the NICHD (R01 HD061504). ELD, $\mathrm{XH}, \mathrm{JV}$, and PT are also supported by NIH grants to PT: U54 EB020403, R01 EB008432, R01 AG040060, and R01 NS080655. CCG is supported by the UCLA BIRC, NS027544, NS05489, Child Neurology Foundation, and the Jonathan Drown Foundation. Scanning was supported by the Staglin IMHRO Center for Cognitive Neuroscience. We gratefully acknowledge the contributions of Alma Martinez and Alma Ramirez in assisting with participant recruitment and study coordination. Finally, the authors thank the participants and their families for contributing their time to this study.

\section{Author Disclosure Statement}

No conflicting financial interests exist.

\section{References}

1. (2015). Concussion Management: Return to Play. www.aap.org/en-us/ advocacy-and-policy/state-advocacy/Documents/concussion.pdf

2. Gogtay, N., Giedd, J.N., Lusk, L., Hayashi, K.M., Greenstein, D., Vaituzis, A.C., Nugent III, T.F., Herman, D.H., Clasen, L.S., Toga, A.W., Rapaport, J.L., and Thompson, P.M. (2004). Dynamic mapping of human cortical development during childhood through early adulthood. Proc. Nat. Acad. Sci. U. S. A. 101, 8174-8179.

3. Dennis, E.L., and Thompson, P.M. (2013). Typical and atypical brain development: a review of neuroimaging studies. Dialogues Clin. Neurosci. 15, 359-383.

4. Sidaros, A., Skimminge, A., Liptrot, M.G., Sidaros, K., Engberg, A.W., Herning, M., Paulson, O.B., Jernigan, T.L., and Rostrup, E. (2009). Long-term global and regional brain volume changes following severe traumatic brain injury: a longitudinal study with clinical correlates. Neuroimage 44, 1-8.

5. Zhou, Y., Kierans, A., Kenul, D., Ge, Y., Rath, J., Reaume, J., Grossman, R.I., and Lui, Y.W. (2013). Mild traumatic brain injury: longitudinal regional brain volume changes. Radiology 267, 880-890.

6. Farbota, K.D., Sodhi, A., Bendlin, B.B., McLaren, D.G., Xu, G., Rowley, H.A., and Johnson, S.C. (2012). Longitudinal volumetric changes following traumatic brain injury: a tensor-based morphometry study. J. Int. Neuropsychol. Soc. 18, 1006-1018.

7. Kim, J., Avants, B., Patel, S., Whyte, J., Coslett, B.H., Pluta, J., Detre, J.A., and Gee, J.C. (2008). Structural consequences of diffuse traumatic brain injury: a large deformation tensor-based morphometry study. Neuroimage 39, 1014-1026.

8. Gale, S.D., Baxter, L., Roundy, N., and Johnson, S.C. (2005). Traumatic brain injury and grey matter concentration: a preliminary voxel based morphometry study. J. Neurol. Neurosurg. Psychiatry 76, 984-988.

9. Yount, R., Raschke, K.A., Biru, M., Tate, D.F., Miller, M.J., Abildskov, T., Gandhi, P., Ryser, D., Hopkins, R.O., and Bigler, E.D. (2002). Traumatic brain injury and atrophy of the cingulate gyrus. J. Neuropsychiatr Clinical Neurosci. 14, 416-423.

10. Tasker, R.C., Salmond, C.H., Westland, A.G., Pena, A., Gillard, J.H., Sahakian, B.J., and Pickard, J.D. (2005). Head circumference and brain and hippocampal volume after severe traumatic brain injury in childhood. Pediatr. Res. 58, 302-308.

11. Wilde, E.A., Bigler, E.D., Hunter, J.V., Fearing, M.A., Scheibel, R.S., Newsome, M.R., Johnson, J.L., Bachevalier, J., Li, X., and Levin, H.S. (2007). Hippocampus, amygdala, and basal ganglia morphometrics in children after moderate-to-severe traumatic brain injury. Dev. Med. Child Neurol. 49, 294-299.

12. Warner, M.A., Youn, T.S., Davis, T., Chandra, A., Marquez de la Plata, C., Moore, C., Harper, C., Madden, C.J., Spence, J., McColl, R., Devous, M., King, R.D., and Diaz-Arrastia, R. (2010). Regionally selective atrophy after traumatic axonal injury. Arch. Neurol. 67, 1336-1344.
13. Himanen, L., Portin, R., Isoniemi, H., Helenius, H., Kurki, T., and Tenovuo, O. (2005). Cognitive functions in relation to MRI findings 30 years after traumatic brain injury. Brain Injury 19, 93-100.

14. Berryhill, P., Lilly, M.A., Levin, H.S., Hillman, G.R., Mendelsohn, D., Brunder, D.G., Fletcher, J.M., Kufera, J., Kent, T.A., Yeakley, J., et al. (1995). Frontal lobe changes after severe diffuse closed head injury in children: a volumetric study of magnetic resonance imaging. Neurosurgery 37, 392-399; discussion 399-400.

15. Levin, H.S., Benavidez, D.A., Verger-Maestre, K., Perachio, N., Song, J., Mendelsohn, D.B., and Fletcher, J.M. (2000). Reduction of corpus callosum growth after severe traumatic brain injury in children. Neurology 54, 647-653.

16. Wilde, E.A., Hunter, J.V., Newsome, M.R., Scheibel, R.S., Bigler, E.D., Johnson, J.L., Fearing, M.A., Cleavinger, H.B., Li, X., Swank, P.R., Pedroza, C., Roberson, G.S., Bachevalier, J., and Levin, H.S. (2005). Frontal and temporal morphometric findings on MRI in children after moderate to severe traumatic brain injury. J. Neurotrauma 22, 333-344.

17. Verger, K., Junque, C., Levin, H.S., Jurado, M.A., Perez-Gomez, M., Bartres-Faz, D., Barrios, M., Alvarez, A., Bartumeus, F., and Mercader, J.M. (2001). Correlation of atrophy measures on MRI with neuropsychological sequelae in children and adolescents with traumatic brain injury. Brain Injury 15, 211-221.

18. Wu, T.C., Wilde, E.A., Bigler, E.D., Li, X., Merkley, T.L., Yallampalli, R., McCauley, S.R., Schnelle, K.P., Vasquez, A.C., Chu, Z., Hanten, G., Hunter, J.V., and Levin, H.S. (2010). Longitudinal changes in the corpus callosum following pediatric traumatic brain injury. Dev. Neurosci. 32, 361-373.

19. Teasdale, G., Murray, G., Parker, L., and Jennett, B. (1979). Adding up the Glasgow coma score, in: Proceedings of the 6th European Congress of Neurosurgery. Vienna, Springer, pps. 13-16.

20. Keenan, H.T., and Bratton, S.L. (2006). Epidemiology and outcomes of pediatric traumatic brain injury. Dev. Neurosci.e 28, 256-263.

21. Dennis, E.L., Jin, Y., Villalon-Reina, J., Zhan, L., Kernan, C., Babikian, T., Mink, R., Babbitt, C., Johnson, J., and Giza, C.C. (2015). White matter disruption in moderate/severe pediatric traumatic brain injury: advanced tract-based analyses. Neuroimage Clin. 7 493-505.

22. Wechsler, D. (2003). Wechsler Intelligence Scale for Children, 4th ed. (WISC-IV). The Psychological Corporation: San Antonio, TX.

23. Delis, D.C., Kramer, J.H., Kaplan, E., and Ober, A. (1994). California Verbal Learning Test-Children's Version (CVLT-C) Manual. Psychological Corporation: San Antonio, TX.

24. Delis, D.C., Kaplan, E., and Kramer, J.H. (2001). Delis-Kaplan Executive Function System (D-KEFS). Psychological Corporation: San Antonio, TX.

25. Babikian, T., and Asarnow, R. (2009). Neurocognitive outcomes and recovery after pediatric TBI: meta-analytic review of the literature. Neuropsychology 23, 283-296.

26. Hua, X., Leow, A.D., Parikshak, N., Lee, S., Chiang, M.-C., Toga, A.W., Jack Jr, C.R., Weiner, M.W., and Thompson, P.M. (2008). Tensor-based morphometry as a neuroimaging biomarker for Alzheimer's disease: an MRI study of $676 \mathrm{AD}, \mathrm{MCI}$, and normal subjects. Neuroimage 43, 458-469.

27. Hua, X., Leow, A.D., Levitt, J.G., Caplan, R., Thompson, P.M., and Toga, A.W. (2009). Detecting brain growth patterns in normal children using tensor-based morphometry. Hum. Brain Mapp. 30, 209-219.

28. Lepore, N., Brun, C., Pennec, X., Chou, Y.Y., Lopez, O.L., Aizenstein, H.J., Becker, J.T., Toga, A.W., and Thompson, P.M. (2007). Mean template for tensor-based morphometry using deformation tensors. Med. Image Comput. Comput. Assist. Interv. 10, 826-833.

29. Leow, A., Huang, S.-C., Geng, A., Becker, J., Davis, S., Toga, A., Thompson, P. (2005). Inverse consistent mapping in 3D deformable image registration: its construction and statistical properties, in: Information Processing in Medical Imaging Christensen and Sonka (eds.). Berlin, Heidelberg, Springer, pps. 493-503.

30. Langers, D.R., Jansen, J.F., and Backes, W.H. (2007). Enhanced signal detection in neuroimaging by means of regional control of the global false discovery rate. Neuroimage 38, 43-56.

31. Babikian, T., Marion, S.D., Copeland, S., Alger, J.R., O’Neill, J., Cazalis, F., Mink, R., Giza, C.C., Vu, J.A., Hilleary, S.M., Kernan, C.L., Newman, N., and Asarnow, R.F. (2010). Metabolic levels in the corpus callosum and their structural and behavioral correlates after moderate to severe pediatric TBI. J. Neurotrauma 27, 473-481. 
32. Marmarou, A. (2007). A review of progress in understanding the pathophysiology and treatment of brain edema. Neurosurg. Focus 22, E1.

33. Ramlackhansingh, A.F., Brooks, D.J., Greenwood, R.J., Bose, S.K., Turkheimer, F.E., Kinnunen, K.M., Gentleman, S., Heckemann, R.A., Gunanayagam, K., Gelosa, G., and Sharp, D.J. (2011). Inflammation after trauma: microglial activation and traumatic brain injury. Ann. Neurol. 70, 374-383.

34. Povlishock, J.T. (2000). Pathophysiology of neural injury: therapeutic opportunities and challenges. Clin. Neurosurg. 46, 113-126.
Address correspondence to:

Emily L. Dennis, PhD

Keck School of Medicine

University of Southern California

2001 N. Soto Street

Los Angeles, CA 90033

E-mail: emily.dennis@ini.usc.edu 